\title{
Dois planos de amostragem para análise de fumonisinas em milho
}

\author{
Two sampling plans for fumonisins analysis in maize
}

\begin{abstract}
Adriano Olnei Mallmann ${ }^{I}$ Alexandro Marchioro' ${ }^{I}$ Maurício Schneider Oliveira ${ }^{I}$ Luciane Minetto Liziane Rachel da Silva Wovst ${ }^{\mathrm{I}}$ Ricardo Hummes Rauber ${ }^{\mathrm{II}}$ Paulo Dilkin ${ }^{\mathrm{I}}$ Carlos Augusto Mallmann ${ }^{\text {* }}$
\end{abstract}

\section{RESUMO}

\begin{abstract}
O objetivo deste trabalho foi avaliar a eficiência de dois planos de amostragem para análise de fumonisinas $\left(B_{1}+B_{2}\right)$ em 11 lotes de milho. Empregaram-se o plano de amostragem manual em grãos inteiros, através do calador graneleiro e o plano de Amostragem Automático em Fluxo Contínuo em grãos moídos (AAFC). Para a comparação dos dois planos de amostragem, foram utilizadas duas medidas de dispersão: a variância e o coeficiente de variação. Em cada plano de amostragem, foram determinadas as variâncias associadas às três etapas do procedimento de detecção das fumonisinas (amostragem, preparação da amostra e análise) e os coeficientes de variação total. A correlação entre as médias de concentrações de fumonisinas e as variâncias de cada etapa do procedimento de detecção foi avaliada para cada plano amostral através da análise de regressão. Na AAFC de milho moído para análise de fumonisinas, obtiveram-se resultados inferiores $(P<0,01)$ de variância na etapa de amostragem $\left(0,0226 \mathrm{mg} \mathrm{kg}^{-1}\right)^{2}$, assim como no coeficiente de variação total $(6,37 \%)$, quando comparados com a variância da amostragem manual $\left(0,0685 \mathrm{mg}^{\mathrm{kg}^{-1}}\right)^{2}$ e coeficiente de variação total do plano de amostragem manual em grãos inteiros (8,94\%), apresentando, portanto, maior eficiência na detecção de fumonisinas em milho.
\end{abstract}

Palavras-chave: micotoxinas, contaminação, homogeneidade, variabilidade, variância, granulometria.

\section{ABSTRACT}

The aim of this research was to evaluate the efficiency of two sampling plans for fumonisins $\left(B_{1}+B_{2}\right)$ analysis in 11 lots of maize. For sample collection the two systems used were a manual system in whole grains, using sampling spear, and an automatic system in milled grains, using the continuous flow sampling system (CFSS). For comparison purpose, two dispersion measurements were used: variance and coefficient of variation. For each sampling plan the variance associated to the three steps of fumonisins quantification (sampling, sample preparation, and analysis), and the total coefficient of variation were determined. The correlation between the average fumonisins concentration and the variances for each phase of the detection procedure was evaluated for each sampling plan using analysis of regression. At the automatic sampling plan in milled grains for fumonisins analysis in maize both sampling variance $\left(0.0226 \mathrm{mg} \mathrm{kg}^{-1}\right)^{2}$ and total coefficient of variation $(6.37 \%)$ were lower $(P<0.01)$ than in the manual sampling plan in whole grains whit $\left(0.0685 \mathrm{mg} \mathrm{kg}^{-1}\right)^{2}$ of variance and $8.94 \%$ of coefficient of variation, showing so better efficiency for fumonisin detection on maize.

Key words: mycotoxin, contamination, homogeneity, variability, variance, granulometry.

\section{INTRODUÇÃO}

As fumonisinas são micotoxinas produzidas por fungos do gênero Fusarium. São contaminantes naturais de grãos e cereais em todo o mundo, principalmente do milho e seus subprodutos (JACKSON \& JABLONSKI, 2004). Segundo LAMIC (2011), nas 26.291 amostras de milho, provenientes principalmente da região Sul do Brasil, analisadas no período de 1996 a 2011, constatou-se a presença de fumonisinas em $76,3 \%$ das amostras, com contaminação

\footnotetext{
'Laboratório de Análises Micotoxicológicas (LAMIC), Departamento de Medicina Veterinária Preventiva (DMVP), Centro de Ciências Rurais (CCR), Universidade Federal de Santa Maria (UFSM). Prédio 44, 97105-000, Camobi, Santa Maria, RS, Brasil. E-mail: mallmann@lamic.ufsm.br. *Autor para correspondência.

"Programa de Pós-graduação em Ciências Veterinárias, Universidade Federal do Rio Grande do Sul (UFRGS), Porto Alegre, RS, Brasil.
} 
média de $1,91 \mathrm{mg} \mathrm{kg}^{-1}$. Nessa região, o milho é o cereal que apresenta o maior índice de contaminação por fumonisinas (MALLMANN \& DILKIN, 2011). A ingestão de alimentos contaminados por fumonisinas pode induzir à leucoencefalomalácia equina (MARASAS et al., 1988) e edema pulmonar em suínos (DILKIN et al., 2004). A Agência Internacional de Pesquisa sobre Câncer classifica a fumonisina $\mathrm{B}_{1}$ como um provável carcinógeno humano (IARC, 2002).

Resultados confiáveis das concentrações de micotoxinas em alimentos são importantes para fins de gerenciamento e controle micotoxicológico. No entanto, é difícil determinar a concentração exata de micotoxinas num lote de cereais ou de alimentos, principalmente em virtude da variabilidade dos resultados, associados aos procedimentos de detecção. WHITAKER et al. (2011) definiram os procedimentos de detecção de micotoxinas como um processo complexo, que consiste de três etapas: 1 retirar uma amostra do lote; 2 - triturar a amostra para reduzir o tamanho das partículas, no caso de amostras em grãos, e dessa retirar uma subamostra para análise; e 3 - extrair a micotoxina da subamostra e quantificá-la.

Considerando-se a distribuição heterogênea das micotoxinas nos cereais, ocorrem variações nos resultados analíticos, denominados de erros. Cada etapa do procedimento de detecção de micotoxinas contém um erro e, consequentemente, não é possível estabelecer o valor real da concentração das micotoxinas em um lote. A eficiência de um procedimento de detecção pode ser medida pela variação em cada uma de suas etapas. A variação total dos resultados da concentração da micotoxina constitui o erro que é o resultado da soma da variância amostral, variância da preparação e variância analítica (OZAY et al., 2006; WHITAKER, 2006; CHELI et al., 2009; WHITAKER et al., 2011).

WHITAKER et al. (1998) avaliaram a variabilidade associada a cada etapa do procedimento de detecção de fumonisinas em lotes de milho. A variabilidade em uma concentração de $2 \mathrm{mg} \mathrm{kg}^{-1}$ de fumonisinas, associada à etapa de amostragem, representou $61 \%$ da variância total dos resultados das concentrações de fumonisinas, enquanto a preparação da amostra e a análise representaram 18,2 e 20,8\%, respectivamente.

Em recente estudo, ANDERSSON et al. (2011) utilizaram dois sistemas de amostragem para detecção de ocratoxina A em cevada e constataram que a amostragem automática, montada posteriormente a uma rosca transportadora, forneceu amostras representativas a partir de um fluxo constante de cevada para detecção de ocratoxina A. Outras alternativas para diminuir a variabilidade dos resultados de contaminação por micotoxinas incluem moer todos os grãos do lote previamente à amostragem, o que aumenta o número de partículas, tornando a distribuição das micotoxinas mais homogênea na massa de alimento, além de aumentar o tamanho da amostra (MIRAGLIA et al., 2005; WHITAKER et al., 2011).

Considerando que existem poucos estudos que descrevem a variação das três etapas do procedimento de detecção de fumonisinas em milho, assim como planos de amostragem para análise de fumonisinas em milho, os objetivos deste trabalho foram comparar a variabilidade (erro) associada a cada etapa do procedimento de detecção de fumonisinas e comparar o coeficiente de variação dos resultados da concentração de fumonisinas entre o plano de amostragem manual em grãos inteiros e o plano de amostragem automático em grãos moídos.

\section{MATERIAL E MÉTODOS}

\section{Coleta das amostras}

A coleta das amostras deste experimento foi realizada na fábrica de rações da Cooperativa Languiru, localizada no município de Estrela, RS, em março de 2010. Foram escolhidos aleatoriamente 11 caminhões com cargas de milho, que variaram de 13 a 32 toneladas. Em cada carga de milho (lote), aplicaram-se os dois planos de amostragem para deteç̧ão de fumonisinas $\mathrm{B}_{1}$ e $\mathrm{B}_{2}$. A amostragem manual foi realizada na massa de grãos inteiros, utilizando-se um calador graneleiro com 10 câmaras coletoras, enquanto a Amostragem Automática em Fluxo Contínuo (AAFC) foi realizada na massa de grãos moídos através de perfurações efetuadas na rosca transportadora. O desenho experimental utilizado para determinar a variabilidade associada a cada etapa do procedimento de detecção de fumonisinas, nos dois planos de amostragem, está ilustrado na figura 1.

No plano de amostragem manual em grãos inteiros, a quantidade de amostra de grãos de milho coletada seguiu as determinações do regulamento EC no 401/2006 da Comissão Europeia. Sendo assim, para lotes com peso de 13 a 20 toneladas, coletaram-se 60 incrementos em 6 pontos de amostragem, totalizando $6 \mathrm{~kg}$, que constituíram a amostra coletiva. Para lotes com mais de 20 toneladas, coletaram-se 100 incrementos em 10 pontos de amostragem, totalizando $10 \mathrm{~kg}$ de amostra coletiva. Esse procedimento de amostragem foi repetido por 10 vezes em cada lote de milho.

No plano de amostragem automático em grãos moídos, os mesmos 11 lotes de milho foram 


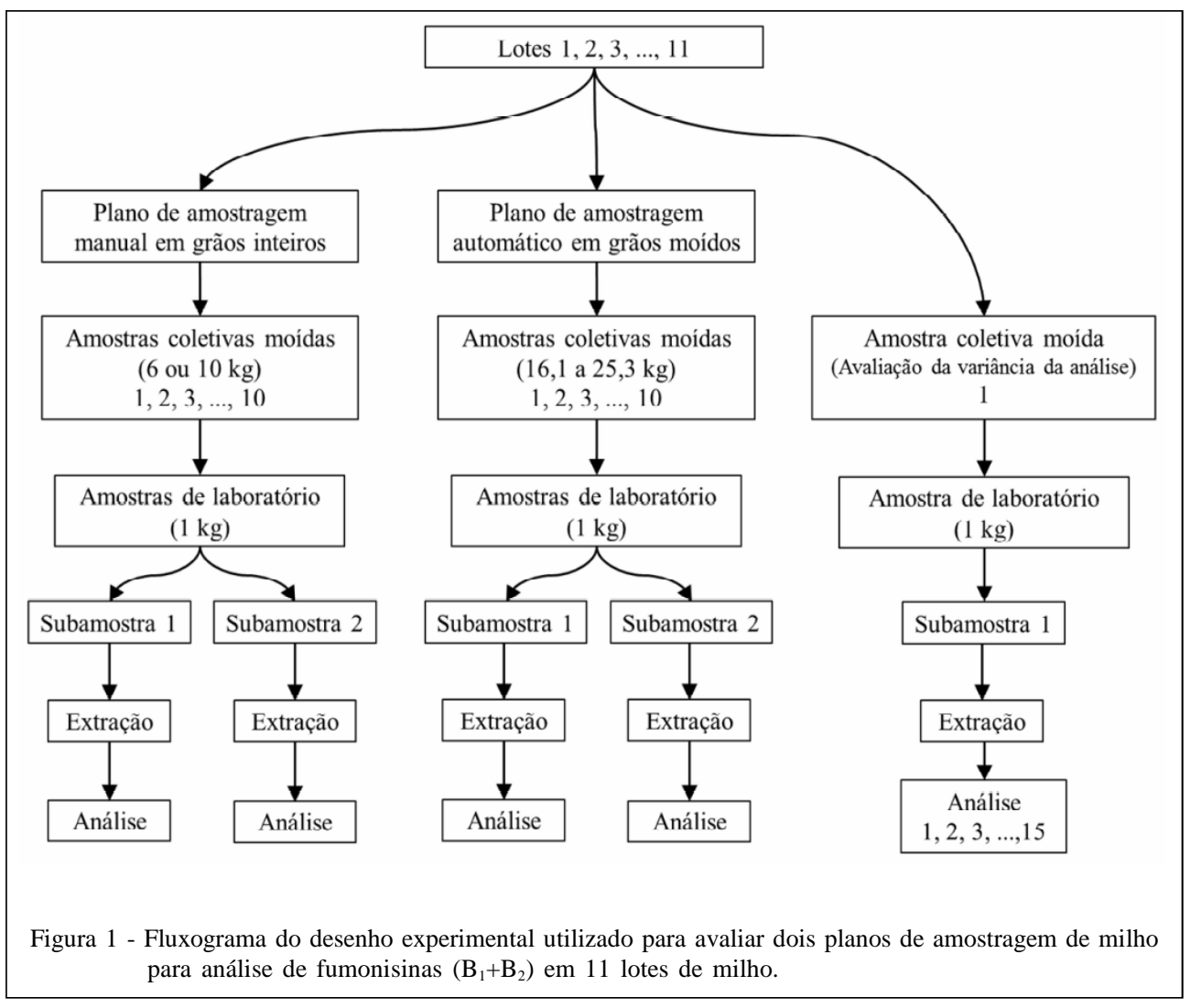

moídos em moinho de martelos com peneira de $3 \mathrm{~mm}$ e movimentados no circuito da fábrica por uma rosca transportadora, também conhecida como caracol ou rosca sem fim. Nessa rosca, com capacidade para transporte de 32 toneladas de farelo de milho por hora, foram instalados 10 sistemas de AAFC para coleta de amostras (Figura 2). Para tanto, foram feitos 10 furos de $17,5 \mathrm{~mm}$ de diâmetro em ângulo de $45^{\circ}$ a partir da base, localizados do lado direito da tubulação externa da rosca transportadora, levando-se em consideração o sentido do fluxo do farelo. Com esse diâmetro, em cada furo, foram coletados incrementos que formaram uma amostra coletiva de peso em $\mathrm{kg}$ igual ao resultado da equação:

Amostra coletiva $=\sqrt{20 * \text { número de toneladas do lote }}$

variando de 16,1 a $25,3 \mathrm{~kg}$ para lotes de 13 a 32 toneladas.

Após o procedimento de coleta, as 20 amostras coletivas de cada lote foram moídas em moinho de martelos com peneira de $2 \mathrm{~mm}$. Em seguida, utilizouse um divisor de amostras de canaletas invertidas (quarteador de amostras) para reduzir as amostras coletivas a $1 \mathrm{~kg}$ (amostras de laboratório), as quais foram embaladas em sacos plásticos e estocadas a $-18^{\circ} \mathrm{C}$.
Preparação das amostras

As amostras de laboratório foram previamente descongeladas à temperatura ambiente por um período de 6 horas. Cada amostra de laboratório foi moída em moinho ultracentrífugo ZM 200 (Retsch, Alemanha), com peneira de $1,5 \mathrm{~mm}$ ( $90 \%$ das partículas com diâmetro inferior a $1 \mathrm{~mm}$ ) e dividida em 10 subamostras, utilizando-se um divisor de amostras PT 100 (Retsch, Alemanha).

Análise das amostras

A análise da presença de fumonisinas $B_{1}+B_{2}$ foi feita em duas subamostras de cada amostra de laboratório. A extração das fumonisinas foi realizada através da adição de $10 \mathrm{~g}$ da subamostra a $50 \mathrm{~mL}$ de solução acetonitrila:água $(1: 1, v / v)$. Em seguida, foram realizadas as seguintes etapas: liquidificação por 5 minutos em blender; filtragem em papel filtro $(14 \mu \mathrm{m})$ para coleta da fração líquida; diluição de $20 \mu \mathrm{L}$ do filtrado em $980 \mu \mathrm{L}$ da solução de diluição acetonitrila:água (1:1, v/v e ácido fórmico 1\%) e análise por Cromatografia

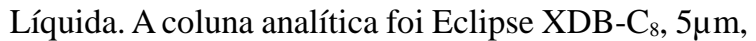
4,6x150mm (Agilent, Brasil) e as fases móveis utilizadas foram água ultrapura e acetonitrila (1:1, v/v e ácido 


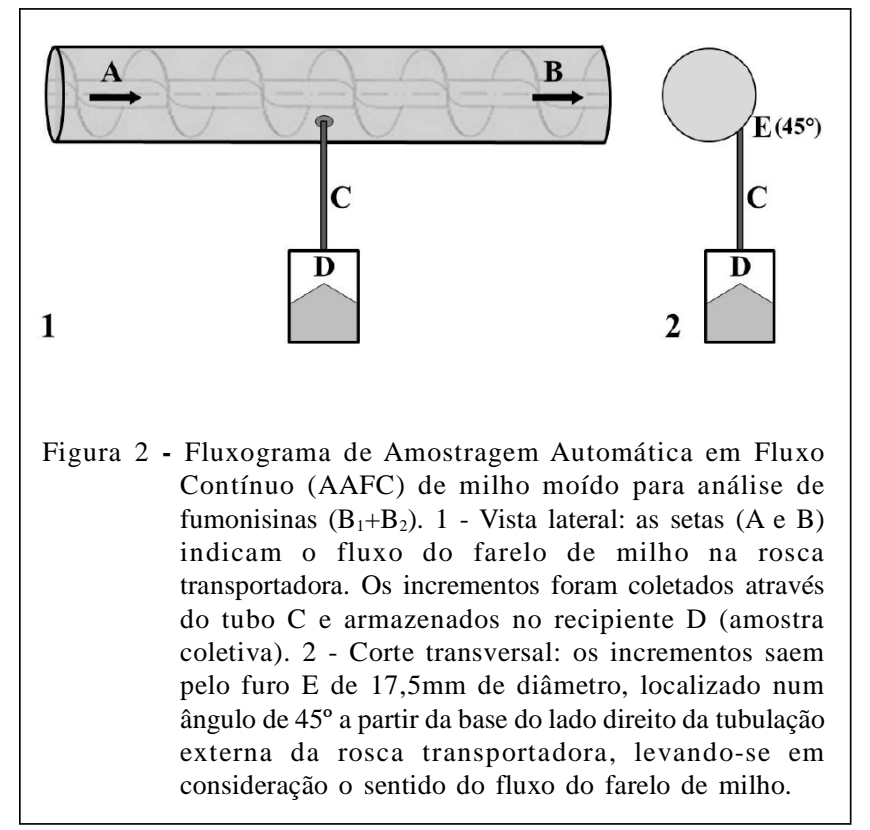

fórmico $0,5 \%$ ). A vazão foi de $0,8 \mathrm{~mL} \mathrm{~min}^{-1}$, com volume de injeção de $5 \mu \mathrm{L}$ e temperatura da coluna de $40^{\circ} \mathrm{C}$. Para a detecção, foi utilizado um espectrômetro de massa sequencial API 5000 (Sciex/Applied Biosystem, Estados Unidos da América) com fonte de ionização por electrospray. As fumonisinas foram quantificadas através de curvas analíticas, preparadas com padrões certificados de fumonisina $\mathrm{B}_{1}$ e $\mathrm{B}_{2}$ (Sigma-Aldrich, Brasil). O método cromatográfico apresentou limite de quantificação de $0,125 \mathrm{mg} \mathrm{kg}^{-1}$. Para o estudo de recuperação, as fortificações foram realizadas em três níveis $\left(0,125,2\right.$ e $\left.10 \mathrm{mg} \mathrm{kg}^{-1}\right)$, com sete replicatas para cada nível de fumonisina $B_{1}$ e $B_{2}$ em três dias diferentes. O percentual de recuperação médio foi de $94,1 \%$ (91,2 a $108,6 \%$ ) para fumonisina $\mathrm{B}_{1}$ e de $94,5 \%$ (93,6 a $\left.108,2 \%\right)$ para fumonisina $B_{2}$.

Determinação da variabilidade associada às três etapas do procedimento de detecção das fumonisinas

A variabilidade dos resultados de detecção das fumonisinas $\left(B_{1}+B_{2}\right)$ foi determinada através do cálculo da variância. A variância total associada ao procedimento de detecção de fumonisinas foi composta pela soma da variância da amostragem, variância da preparação e variância da análise, conforme a equação:

Variância total $=$ Variância da amostragem + Variância da preparação + Variância analítica

Obteve-se a variância da amostragem, variância da preparação e variância da análise em cada lote e plano de amostragem através dos resultados observados para o lote (10 amostras de laboratório), amostra de laboratório (duas subamostras) e extrato (15 alíquotas), respectivamente. A variância da análise foi calculada utilizando somente um extrato de cada lote para ambos os planos de amostragem. No total, foram realizadas 1.210 análises de fumonisinas $\left(\mathrm{B}_{1} \mathrm{e} \mathrm{B}_{2}\right)$.

Análise estatística

As variâncias de cada etapa do procedimento de detecção e os CV (\%) de cada lote foram submetidos à análise de variância multifatorial, utilizando a contaminação média de fumonisinas em cada variância como covariável, e comparando as variâncias pelo teste $\mathrm{F}$ a $5 \%$ de significância. As variâncias da amostragem para cada plano amostral, bem como as variâncias de preparação e análise (independente do plano amostral utilizado) foram submetidas à análise de regressão, em relação às médias de contaminação por fumonisinas $\left(\mathrm{B}_{1}+\mathrm{B}_{2}\right)$, para elaboração das equações. Os modelos utilizados foram os que apresentaram menor valor de P. Os dados foram analisados no programa estatístico Statgraphics Centurion XV, versão 15.2.11.

\section{RESULTADOS E DISCUSSÃO}

A média de concentração de fumonisinas, $o$ coeficiente de variação, as variâncias associadas as três etapas e a variância total, nos dois planos de amostragem, por lote de milho, estão representados na tabela 1. Os resultados da análise de regressão (Tabela 2) indicam que cada componente da variância total 
Tabela 1 - Média de concentração de fumonisinas $\left(\mathrm{mg} \mathrm{kg}^{-1}\right)$ e coeficiente de variação (\%) dos resultados das concentrações de fumonisinas, no plano de amostragem manual em grãos inteiros e no plano de amostragem automático em grãos moídos, por lote de milho, com as respectivas variâncias, associadas às etapas de amostragem, preparação de amostras, análise e variância total.

\begin{tabular}{|c|c|c|c|c|c|c|c|}
\hline \multirow{2}{*}{ Lote } & \multirow{2}{*}{ Plano de amostragem } & \multirow{2}{*}{ Média $\left(\mathrm{mg} \mathrm{kg}^{-1}\right)$ Fumonisinas* } & \multirow{2}{*}{$\mathrm{CV}(\%)^{* *}$} & \multicolumn{4}{|c|}{ - Variância $\left(\mathrm{mg} \mathrm{kg}^{-1}\right)^{2}$} \\
\hline & & & & Amostragem & Preparação & Análise & Total \\
\hline 1 & Manual & 2,73 & 8,10 & 0,0341 & 0,0094 & 0,0052 & 0,0488 \\
\hline 2 & Manual & 2,02 & 11,12 & 0,0391 & 0,0054 & 0,0061 & 0,0506 \\
\hline 3 & Manual & 2,53 & 11,33 & 0,0678 & 0,0072 & 0,0071 & 0,0821 \\
\hline 4 & Manual & 6,29 & 6,40 & 0,1027 & 0,0332 & 0,0269 & 0,1629 \\
\hline 5 & Manual & 5,57 & 9,29 & 0,1655 & 0,0907 & 0,0112 & 0,2674 \\
\hline 6 & Manual & 2,98 & 9,04 & 0,0639 & 0,0001 & 0,0087 & 0,0726 \\
\hline 7 & Manual & 3,45 & 8,87 & 0,0574 & 0,0305 & 0,0058 & 0,0936 \\
\hline 8 & Manual & 2,47 & 9,67 & 0,0482 & 0,0014 & 0,0074 & 0,0570 \\
\hline 9 & Manual & 5,13 & 6,48 & 0,0853 & 0,0135 & 0,0115 & 0,1103 \\
\hline 10 & Manual & 2,63 & 9,62 & 0,0476 & 0,0130 & 0,0035 & 0,0642 \\
\hline 11 & Manual & 3,35 & 8,09 & 0,0496 & 0,0152 & 0,0087 & 0,0736 \\
\hline 1 & Automático & 2,77 & 6,87 & 0,0223 & 0,0085 & 0,0052 & 0,0360 \\
\hline 2 & Automático & 1,76 & 7,83 & 0,0099 & 0,0029 & 0,0061 & 0,0189 \\
\hline 3 & Automático & 2,76 & 7,44 & 0,0247 & 0,0103 & 0,0071 & 0,0421 \\
\hline 4 & Automático & 6,92 & 6,90 & 0,0435 & 0,1587 & 0,0269 & 0,2292 \\
\hline 5 & Automático & 4,05 & 4,88 & 0,0140 & 0,0138 & 0,0112 & 0,0390 \\
\hline 6 & Automático & 2,59 & 7,72 & 0,0218 & 0,0097 & 0,0087 & 0,0401 \\
\hline 7 & Automático & 3,80 & 5,91 & 0,0341 & 0,0106 & 0,0058 & 0,0505 \\
\hline 8 & Automático & 2,60 & 7,09 & 0,0224 & 0,0041 & 0,0074 & 0,0339 \\
\hline 9 & Automático & 4,73 & 5,14 & 0,0314 & 0,0162 & 0,0115 & 0,0591 \\
\hline 10 & Automático & 2,85 & 5,84 & 0,0098 & 0,0144 & 0,0035 & 0,0277 \\
\hline 11 & Automático & 3,26 & 4,70 & 0,0072 & 0,0076 & 0,0087 & 0,0235 \\
\hline
\end{tabular}

* Média dos resultados de fumonisinas $\left(\mathrm{B}_{1}+\mathrm{B}_{2}\right)$ das 20 subamostras analisadas.

** CV: Coeficiente de variação $(\%)$.

aumentou $(r>0,83)$ com o aumento da concentração de fumonisinas. Essa característica também foi observada por WHITAKER et al. (1998) em estudo com fumonisinas em milho e por estudos realizados com aflatoxinas em sementes de algodão (WHITAKER et al., 1976), milho (WHITAKER et al., 1979), amendoim (WHITAKER et al., 1994) e amêndoas (WHITAKER et al., 2006).

A maior fonte de variação dos resultados das concentrações de fumonisinas para ambos os planos de amostragem foi na etapa de amostragem, sendo que as variâncias foram três vezes menores $(\mathrm{P}<0,01)$ para a amostragem automática em grãos moídos em relação à amostragem manual em grãos inteiros (Tabela 2). Pesquisadores concluíram que a etapa de amostragem é a maior fonte de variabilidade associada ao procedimento de detecção de: fumonisinas em milho (WHITAKER et al., 1998, 2007); aflatoxinas em milho (WHITAKER et al., 1979; JOHANSSON et al., 2000); aflatoxinas em amendoim (WHITAKER et al., 1994); aflatoxinas em avelã (OZAY et al., 2006), deoxinivalenol e ocratoxina A em trigo (BISELLI et al., 2008).
As amostras utilizadas neste estudo apresentaram resultados de concentrações de fumonisinas de 1,55 a $7,45 \mathrm{mg} \mathrm{kg}^{-1}$. Os valores do Desvio Padrão Relativo de repetibilidade (DPRr) dos resultados das concentrações de fumonisinas $\left(\mathrm{B}_{1}+\mathrm{B}_{2}\right)$ das duas subamostras analisadas, provenientes de cada amostra de laboratório, variou de 0,03 a 17,8\%. O valor máximo aceitável de DPRr, estabelecido pelo regulamento EC $\mathrm{N}^{\circ} 401 / 2006$, para contaminações superiores a $0,5 \mathrm{mg}$ $\mathrm{kg}^{-1}$ de $\mathrm{B}_{1}+\mathrm{B}_{2}$ é de $20 \%$. Isso demonstra que os procedimentos de preparação das amostras e análise obtiveram valores aceitáveis de DPRr.

Neste trabalho, foi assumido que as diferenças observadas nos valores do $\mathrm{CV}$ entre as amostras de cada lote estavam associadas ao procedimento de amostragem, uma vez que os procedimentos de preparo das amostras e de análise empregados foram os mesmos. Os resultados dos CV das concentrações de fumonisinas dos 11 lotes indicam que o CV da amostragem automática, com média de $6,37 \%$, foi menor $(\mathrm{P}<0,01)$ que o $\mathrm{CV}$ da amostragem manual, com média de $8,94 \%$. 
Tabela 2 - Média das variâncias em cada etapa do procedimento de detecção de fumonisinas (amostragem, preparação e análise), no plano de amostragem manual em grãos inteiros e no plano de amostragem automático em grãos moídos, e equações de regressão das variâncias em relação à média de contaminação por fumonisinas em cada lote de milho.

\begin{tabular}{|c|c|c|c|}
\hline \multirow{2}{*}{ Etapa } & ----------Variâncias do & tragem $\left(\mathrm{mg} \mathrm{kg}^{-1}\right)^{2}$ & \multirow{2}{*}{$\mathrm{P}$} \\
\hline & Manual em grãos inteiros & Automático em grãos moídos & \\
\hline Amostragem & $0,0685^{\mathrm{a}}$ & $0,0226^{\mathrm{b}}$ & 0,00 \\
\hline Preparação & $0,0190^{\mathrm{a}}$ & $0,0243^{\mathrm{a}}$ & 0,62 \\
\hline Análise & $0,0091^{\mathrm{a}}$ & $0,0095^{\mathrm{a}}$ & 0,80 \\
\hline Total & $0,0967^{\mathrm{a}}$ & $0,0563^{\mathrm{b}}$ & 0,01 \\
\hline Equação amostragem manual & \multicolumn{3}{|c|}{ Variância da Amostragem $=2,718281828^{\left(-4,01392+1,02862^{*} \operatorname{LN}(X)\right)} \quad(\mathrm{P}<0,01 ; \mathrm{r}=0,836)$} \\
\hline Equação amostragem automática & \multicolumn{3}{|c|}{ Variância da Amostragem $=\sqrt{ }\left(0,0000823063+0,0000373211 * X^{2}\right) \quad(P<0,01 ; r=0,835)$} \\
\hline Equação Preparação & \multicolumn{3}{|c|}{ Variância da Preparação $=\left(0,0382133+0,00589541 * X^{2}\right)^{2}(P=0 ; r=0,835)$} \\
\hline Equação Análise ${ }^{\#}$ & \multicolumn{3}{|c|}{ Variância da Análise $=0,00271687+0,00046355^{*} \mathrm{X}^{2}(\mathrm{p}=0 ; \mathrm{r}=0,900)$} \\
\hline
\end{tabular}

${ }^{\mathrm{a}-\mathrm{b}}$ Médias seguidas de letras iguais em cada linha não diferem entre os planos de amostragem pelo teste $\mathrm{F}(\mathrm{P} \leq 0,05)$. Médias das variâncias dos 11 lotes.

LN: Logaritmo Natural.

$\mathrm{X}$ : Média de contaminação por fumonisinas $\mathrm{B}_{1}+\mathrm{B}_{2}\left(\mathrm{mg} \mathrm{kg}^{-1}\right)$.

\# Procedimentos de preparo da amostra e análise foram iguais nos dois planos de amostragem.

SHOTWELL et al. (1975) constataram que, se um lote é bem misturado previamente, as partículas contaminadas provavelmente são distribuídas uniformemente por toda sua massa. Nessa situação, o local do qual a amostra é retirada do lote, provavelmente não é muito importante. No entanto, se o lote não for misturado previamente, as partículas contaminadas com micotoxinas podem estar localizadas em áreas delimitadas. Sendo assim, se o plano amostral for realizado coletando amostras a partir de um único ponto do lote, as partículas contaminadas podem não ser coletadas, ou, ainda, muitas partículas contaminadas podem ser coletadas (MALLMANN \& DILKIN, 2011), situações estas que podem ocorrer com maior intensidade no plano de amostragem manual em grãos inteiros, abordado neste estudo.

A representatividade da coleta de uma amostra é maior se ela for efetuada em materiais moídos, pelo maior número de partículas amostradas por unidade de volume ou unidade de massa, em comparação a grãos inteiros (TITTLEMIER et al., 2011). O tamanho da amostra interfere na variabilidade dos resultados, ou seja, quanto maior a amostra, menor a variação (WHITAKER et al., 2011). A coleta de incrementos enquanto o lote é movido de um local para outro (lote dinâmico), confere melhor representatividade à amostra, quando comparada à amostragem efetuada de um lote estático (MIRAGLIA et al., 2005; WHITAKER, 2006; MALLMANN \& DILKIN, 2011). Portanto, a redução da variabilidade dos resultados das concentrações de fumonisinas na AAFC neste experimento provavelmente está associada ao fato de que todo o lote é moído e, em seguida, conduzido por uma rosca transportadora, de modo que, tanto a moagem quanto a rosca transportadora auxiliam na homogeneização do lote. Além disso, o tamanho da amostra coletiva do plano de amostragem automático em grãos moídos é maior e a coleta de incrementos é ininterrupta, ou seja, sempre há uma pequena fração sendo coletada, possibilitando uma melhor aleatoriedade na coleta das partículas e, consequentemente, melhor representatividade da amostra.

\section{CONCLUSÃO}

As variâncias (erros) associadas ao procedimento de detecção das fumonisinas nas etapas de amostragem, preparação da amostra e análise aumentam com o aumento da concentração de fumonisinas no milho, indiferente do plano de amostragem utilizado. No entanto, o plano de amostragem automático, por ser aplicado em grãos moídos em movimento, de forma contínua e com amostra coletiva maior, reduz a variância associada à etapa de amostragem e à variância total, em comparação ao plano de amostragem manual em grãos inteiros. Constatou-se também que o CV das concentrações de fumonisinas do plano de amostragem automático em grãos moídos é menor que o CV do plano de amostragem manual em grãos inteiros. 


\section{AGRADECIMENTOS}

À Cooperativa Languiru, por ter disponibilizado suas instalações para a coleta das amostras de milho; à Coordenação de Aperfeiçoamento de Pessoal de Nível de Superior (CAPES), pela concessão de bolsa de estudos ao primeiro autor; e à Fundação de Amparo à Pesquisa do Estado do Rio Grande do Sul (FAPERGS), pelo apoio financeiro.

\section{REFERÊNCIAS}

ANDERSSON, M.G et al. Comparison of manual and automatic sampling for ochratoxin A in barley grain. Food Additives and Contaminants: part A, v.28, n.8, p.1066-1075, ago. 2011. Disponível em: 〈http://dx.doi.org/10.1080/19440049.2011.576438〉. Acesso em: 24 jun. 2011. doi: 10.1080/19440049.2011.576438.

BISELLI, S. et al. Investigation of the distribution of deoxynivalenol and ochratoxin A contamination within a $26 \mathrm{t}$ truckload of wheat kernels. Mycotoxin Research, v.24, n.2, p.98-104, jun. 2008. Disponível em: <http://www.springerlink.com/content/ c129160051046131/>. Acesso em: 24 jun. 2011. doi: 10.1007/ BF02985287.

CHELI, F. et al. Sampling feed for mycotoxins: acquiring knowledge from food. Italian Journal of Animal Science, v.8, p.5-22, dez. 2009. Disponível em: <http://www.aspajournal.it/ index.php/ijas/article/view/24>. Acesso em 24 jun. 2011. doi: 10.4081/ijas.2009.5.

DILKIN, P. et al. Intoxicação experimental de suínos por fumonisinas. Ciência Rural, v.34, n.1, p.175-181, jan./fev. 2004. Disponível em: <http://www.scielo.br/scielo.php?script=sci_arttext\&pid=S0103$84782004000100027 \& \operatorname{lng}=$ en\&nrm=iso $>$. Acesso em: 24 jun. 2011. doi: $10.1590 / \mathrm{S} 0103-84782004000100027$.

EUROPEAN COMMISSION. Commission Regulation EC n0ㅇ/ 2006 of 23 February 2006 laying down the methods of sampling and analysis for the official control of the levels of mycotoxins in foodstuffs. Official Journal of the European Union, L 70/12. Disponível em: <http://eur-lex.europa.eu/LexUriServ/ LexUriServ.do?uri=CELEX:32006R0401:en:NOT>.Acesso em: 09 fev. 2010.

IARC (INTERNATIONAL AGENCY FOR RESEARCH ON CANCER). Fumonisin $B_{1}$. some traditional herbal medicines, some mycotoxins, naphthalene and styrene. Monographs on the Evaluation of Carcinogenic Risks to Humans, v. 82, p.301-366, dez. 2002. Disponível em: <http://monographs.iarc.fr/ ENG/Monographs/vol82/mono82.pdf>. Acesso em: 04 jul. 2011.

JACKSON, L.; JABLONSKI, J. Fumonisins. In: MAGAN, N.; OLSEN, M. Mycotoxins in food. Cambridge: Woodhead, 2004. Cap.16, p.367-391.

JOHANSSON, A.S. et al. Testing shelled corn for aflatoxin, part I: estimation of variance components. Journal of AOAC International, v.83, n.5, p.1264-1269, set./out. 2000. Disponível em: 〈http://www.bae.ncsu.edu/usda/www/researchpaperstbw.htm〉. Acesso em: 17 fev. 2010.

LAMIC (LABORATÓRIO DE ANÁLISES MICOTOXICOLÓGICAS), Universidade Federal de Santa Maria, Santa Maria, RS, Brasil. Tabelas de resultados, 2011. Disponível em: 〈http://www.lamic.ufsm.br〉. Acesso em: 21 dez. 2011.
MALLMANN, C.A.; DILKIN, P. Mycotoxins and mycotoxicosis in swine. Coconut Grove: Special Nutrients, 2011. 182p.

MARASAS, W.F. et al. Leukoencephalomalacia in a horse induced by fumonisin B isolated from Fusarium moniliforme. Onderstepoort Journal of Veterinary Research, v.55, n.4, p.197-203, dez. 1988. Disponível em: <http://www.ncbi.nlm.nih.gov/ pubmed/3217091>. Acesso em: 20 abr. 2011.

MIRAGLIA, M. et al. The role of sampling in mycotoxin contamination: an holistic view. Food Additives and Contaminants: part A, v.22, n.1, p.31-36, jan. 2005. Disponível em: <http://dx.doi.org/10.1080/02652030500389055>. Acesso em: 20 jul. 2010. doi: 10.1080/02652030500389055.

OZAY, G. et al. Sampling hazelnuts for aflatoxin: uncertainty associated with sampling, sample preparation, and analysis. Journal of AOAC International, v.89, n.4, p.1004-1011, jul./ago. 2006. Disponível em: <http://www.bae.ncsu.edu/usda/ www/x1pub/paper111.pdf>. Acesso em: 17 fev. 2010.

SHOTWELL, O.L. et al. Mycotoxins in hot spots in grains. 1. Aflatoxin and zearalenone occurrence in stored corn. Cereal Chemistry, v.52, n.5, p.687-697, set./out. 1975. Disponível em: <http://ddr.nal.usda.gov/bitstream/10113/29962/1/ CAIN759100009.pdf $>$. Acesso em: 16 jun. 2011.

TITTLEMIER, S.A. et al. Sampling of cereals and cerealbased foods for the determination of ochratoxin A: an overview. Food Additives and Contaminants: Part A, v.28, n.6, p.775778, jun. 2011. Disponível em: <http://www.tandfonline.com/ doi/pdf/10.1080/19440049.2011.559278>. Acesso em: 24 nov. 2011. doi: 10.1080/19440049.2011.559278.

WHITAKER, T.B. et al. Variability associated with testing cottonseed for aflatoxin. Journal of the American Oil Chemists' Society, v.53, n.7, p.502-505, jul. 1976. Disponível em: <http://www.bae.ncsu.edu/usda/www/ researchpaperstbw.htm>. Acesso em: 17 fev. 2010.

WHITAKER, T.B. et al. Variability associated with testing corn for aflatoxin. Journal of the American Oil Chemists' Society, v.56, n.9, p.789-794, set. 1979. Disponível em: <http:/ /www.bae.ncsu.edu/usda/www/researchpaperstbw.htm>. Acesso em: 17 fev. 2010.

WHITAKER, T.B. et al. Variability associated with sampling, sample preparation, and chemically testing for aflatoxin in farmers' stock peanuts. Journal of AOAC International, v.77, n.1, p.107-116, jan./fev. 1994. Disponível em: <http:// www.bae.ncsu.edu/usda/www/researchpaperstbw.htm>. Acesso em: 17 fev. 2010

WHITAKER, T.B. et al. Variability associated with testing shelled corn for fumonisin. Journal of AOAC International, v.81, n.6, p.1162-1168, nov./dez. 1998. Disponível em: 〈http:/ /www.bae.ncsu.edu/usda/www/researchpaperstbw.htm>. Acesso em: 10 abr. 2010.

WHITAKER, T.B. Sampling food for mycotoxins. Food Additives and Contaminants, v.23, n.1, p.50-61, jan. 2006. Disponível em: <http://www.ncbi.nlm.nih.gov/pubmed/ 16393815>. Acesso em: 17 ago. 2010. doi: 10.1080/ 02652030500241587 . 
WHITAKER, T.B. et al. Sampling almonds for aflatoxin, part I: estimation of uncertainty associated with sampling, sample preparation, and analysis. Journal of AOAC International, v.89, n.4, p.1027-1034, jul./ago. 2006. Disponível em: <http:// www.ncbi.nlm.nih.gov/pubmed/16915840>. Acesso em: 17 fev. 2010.

WHITAKER, T.B. et al. Evaluating the performance of sampling plans to detect fumonisin $B_{1}$ in maize lots marketed in Nigeria.
Journal of AOAC International, v.90, n.4, p.1050-1059, jul./ago. 2007. Disponível em: <http://www.ncbi.nlm.nih.gov/ pubmed/17760343>. Acesso em: 17 fev. 2010.

WHITAKER, T.B. et al. Sampling procedures to detect mycotoxins in agricultural commodities. New York: Springer, 2011. 58p. 